\title{
Boundary state from open string field theory invariants
}

\section{Carlo Maccaferri ${ }^{* \dagger}$}

Dipartimento di Fisica, Universitá di Torino and INFN Sezione di Torino

Via Pietro Giuria 1, I-10125 Torino, Italy

E-mail: maccafer at gmail.com

In this talk I show how, given a solution of open string field theory, it is possible to simply compute the associated boundary state by evaluating the coefficients in front of the Ishibashi states from a generalization of well known gauge invariant observables. After outlining the general method, I give explicit constructions from analytic and numerical solutions.

Proceedings of the Corfu Summer Institute 2012 "School and Workshops on Elementary Particle Physics and Gravity"

September 8-27, 2012

Corfu, Greece

\footnotetext{
*Speaker.

${ }^{\dagger}$ This talk is based on a collaboration with Matej Kudrna and Martin Schnabl, [B]], which I warmly thank
} 


\section{Introduction}

Open string field theory (OSFT), [四] is a useful and in-principle-complete framework to explore important aspects of the non perturbative structure of string theory. It was originally constructed as a second quantized formulation for the open string in order to derive string amplitudes from 'space-time' Feynmann diagrams. It is a classical result that the collection of Feynmann diagrams (in Siegel gauge) gives a single and unique covering of the moduli space of Riemann surfaces with boundaries, open string punctures and closed string punctures, [园]. Therefore all open-closed string amplitudes, at any order in perturbation theory, can be obtained from open string field theory. Being a second quantized formulation for the open strings we can, in addiction, derive consistent extensions of the amplitudes where open strings (but not closed strings) can be taken off-shell.

While the possibility to go off shell is not particularly useful at the perturbative level, it allows to move out from the perturbative vacuum, where the theory is initially defined, to different backgrounds with different excitations. Since open strings are excitations of D-branes, it is natural to expect that classical solutions to the equation of motion of open string field theory should correspond to different D-branes configurations which can be consistently placed in a given closed string background. While all classical solutions we know of (as of today) are consistent with this expectation, it is in general not easy to understand the way OSFT encodes the D-brane moduli in terms of the parameters of the corresponding classical solutions. A related problem is that the huge gauge invariance of OSFT can connect seemingly different solutions and it is in general hard to distinguish between physical degrees of freedom and gauge redundant one. We thus seek for a tool which allows to describe the physical property of the D-brane system the solution is describing, in a gauge invariant way.

At the world sheet level, the most accurate description of a D-brane system, is its boundary state $\left|B_{*}\right\rangle$. A boundary state is a closed string state which, when overlapped with generic closed string states, gives as a result the disk tadpoles of the latter, with the boundary conditions of a given boundary conformal field theory $\mathrm{BCFT}_{*}$, which $\left|B_{*}\right\rangle$ is encoding. It should then be possible to re-organize the gauge invariant data of a given OSFT solution and recast them in the form of the boundary state the solution is describing. This is what I aim at describing in this contribution.

\section{Construction}

Let $\Psi$ be a solution to the OSFT equation of motion

$$
Q \Psi+\Psi * \Psi=0
$$

Our aim is to compute the boundary state, $|B \Psi\rangle$, which corresponds to the boundary conditions which the solution is describing. We assume that the boundary state is a ghost number 3 closed string state which describes conformal boundary conditions in the total $c=0$ matter/ghost CFT. At the disk level this means

$$
\begin{aligned}
\left(b_{0}-\bar{b}_{0}\right)\left|B_{\Psi}\right\rangle & =0 \\
Q_{g h}\left|B_{\Psi}\right\rangle & =3\left|B_{\Psi}\right\rangle \\
\left(L_{n}-\bar{L}_{-n}\right)\left|B_{\Psi}\right\rangle & =0 .
\end{aligned}
$$


As shown in [3] the above constraints are enough to guarantee that the boundary state $\left|B_{\Psi}\right\rangle$ is matter ghost factorized

$$
\left|B_{\Psi}\right\rangle=\left|B_{\Psi}\right\rangle^{\text {matter }} \otimes\left|B_{g h}\right\rangle
$$

and that

$$
\begin{aligned}
\left(b_{n}-\bar{b}_{-n}\right)|B \Psi\rangle & =0, \\
\left(c_{n}+\bar{c}_{-n}\right)|B \Psi\rangle & =0, \\
\left(L_{n}^{\text {matter }}-\bar{L}_{-n}^{\text {matter }}\right)|B \Psi\rangle & =0, \\
Q_{B R S T}\left|B_{\Psi}\right\rangle & =0 .
\end{aligned}
$$

As it is well known, the Virasoro gluing condition in the matter sector implies that the generic form of the matter part of the boundary state is a linear combination of Ishibashi states, [[]]. Let $\left\{V_{\alpha}\right\}$ be the collection of non-singular spinless bulk primaries of weight $\left(h_{\alpha}, h_{\alpha}\right)$ in the matter $\mathrm{CFT}^{1}$

$$
\begin{aligned}
\left(L_{0}-\bar{L}_{0}\right)\left|V_{\alpha}\right\rangle & =\left(h_{\alpha}-h_{\alpha}\right)\left|V_{\alpha}\right\rangle=0 \\
L_{n}\left|V_{\alpha}\right\rangle & =\bar{L}_{n}\left|V_{\alpha}\right\rangle=0, \quad n \geq 1 .
\end{aligned}
$$

Let's define a BPZ-dual basis of primaries $\left\{V^{\beta}\right\}$ such that

$$
\left\langle V^{\alpha} \mid V_{\beta}\right\rangle=\delta_{\beta}^{\alpha} .
$$

This is possible once singular (null) states have been projected out. To any spinless vertex operator $V_{\alpha}$ we can associate the corresponding conformal Ishibashi state, which (up to normalization) is the unique state $\left.\left|V_{\alpha}\right\rangle\right\rangle$ in the Virasoro Verma module of $V_{\alpha}$ satisfying the Virasoro gluing conditions

$$
\left.\left(L_{n}-\bar{L}_{-n}\right)\left|V_{\alpha}\right\rangle\right\rangle=0 .
$$

A simple closed form of the solution to the gluing condition for the general case can be written as

$$
\left.\left|V_{\alpha}\right\rangle\right\rangle=\sum_{n}|n, \alpha\rangle \otimes \overline{|n, \alpha\rangle},
$$

where the sum runs over orthonormal basis of states in the irreducible representation of the chiral Virasoro algebra built over the primary $V_{\alpha}$. The normalization has been chosen so that

$$
\left.\left\langle V^{\alpha} \mid V_{\beta}\right\rangle\right\rangle=\left\langle V^{\alpha} \mid V_{\beta}\right\rangle=\delta_{\beta}^{\alpha} .
$$

The matter part of our to-be-found boundary state $\left|B_{\Psi}\right\rangle$ can thus be written as

$$
\left.|B \Psi\rangle^{\text {matter }}=\sum_{\alpha} n_{\Psi}^{\alpha}\left|V_{\alpha}\right\rangle\right\rangle
$$

Therefore, the only needed informations to construct the boundary state are the coefficients $n_{\Psi}^{\alpha}$. These coefficients are nothing but the disk one point functions of the spinless primaries in the matter $\mathrm{BCFT}_{\Psi}$ with the new boundary conditions implicitly described by the solution $\Psi$

$$
n_{\Psi}^{\alpha}=\left\langle V^{\alpha} \mid B \Psi\right\rangle^{\text {matter }} \equiv\left\langle V^{\alpha}(0)\right\rangle_{\text {disk }}^{\mathrm{BCFT}_{\Psi}^{\text {matter }}}
$$

\footnotetext{
${ }^{1}$ In CFTs on noncompact target spaces $\alpha$ will in general be a continuous variable, like the momentum.
} 
Our aim is to compute these coefficients from gauge invariant observables of OSFT.

Suppose, for a moment, that we want to compute $n_{\Psi}^{\alpha}$ for a bulk primary of weight 1 . To do so, we have at our disposal a very well known OSFT invariant, [四, [1, 回, 四]

$$
W_{V}(\Psi) \equiv\langle I|\mathscr{V}(i)| \Psi\rangle
$$

where $\langle I|$ is the identity string field and $\mathscr{V}(i)$ is a ghost number two closed string vertex operator, placed at the midpoint. This object is well defined and gauge invariant when the midpoint insertion $\mathscr{V}(i)$ is a BRST closed weight-zero primary. Let us take

$$
\mathscr{V}=c \bar{c} V_{h=1}^{\text {matter }}
$$

where $V_{h=1}^{\text {matter }}$ is a spinless weight 1 primary in the matter CFT. Then, based on the available examples, Ellwood coinjectured $[\mathbb{}]$ that this invariant is precisely computing the shift in the closed string tadpole from the starting $\mathrm{BCFT}_{0}$ to the target $\mathrm{BCFT}_{\Psi}$, described by the solution $\Psi$.

$$
\langle I|\mathscr{V}(i)| \Psi\rangle=\frac{1}{2 \pi i}\langle\mathscr{V}(0) c(1)\rangle_{\mathrm{disk}}^{\mathrm{BCFT}_{0}}-\frac{1}{2 \pi i}\langle\mathscr{V}(0) c(1)\rangle_{\mathrm{disk}}^{\mathrm{BCFT} \Psi}
$$

We can rewrite this relation using the fact that the invariant of the tachyon vacuum $\Psi_{T V}$ should precisely account for the $\mathrm{BCFT}_{0}$ contribution

$$
\left\langle I|\mathscr{V}(i)| \Psi-\Psi_{T V}\right\rangle=-\frac{1}{2 \pi i}\langle\mathscr{V}(0) c(1)\rangle_{\mathrm{disk}}^{\mathrm{BCFT} \Psi}
$$

If we now explicitly split the disk tadpole in matter and ghost, we get from the ghost part

$$
\langle c \bar{c}(0) c(1)\rangle_{\text {disk }}=-1,
$$

and we thus find

$$
n_{\psi}^{h=1}=\left\langle V_{h=1}^{\text {matter }}(0)\right\rangle_{\text {disk }}^{\mathrm{BCFT}_{\Psi}^{\text {matter }}}=2 \pi i\left\langle I|\mathscr{V}(i)| \Psi-\Psi_{T V}\right\rangle .
$$

Therefore the Ellwood invariants compute the coefficients of the Ishibashi states of the weight one bulk primaries.

Our aim is to generalize these invariants in such a way that they can compute the tadpoles for generic matter primaries. To do this we describe the system 'from outside', so that we can compensate the excess of weight in the closed string primary in an auxiliary direction. We tensor our initial $\mathrm{BCFT}_{0}$ with a properly chosen $c=0$ auxiliary BCFT ${ }^{\text {aux }}$ and search for a lifted solution $\tilde{\Psi}$, to the equation of motion

$$
\tilde{Q} \tilde{\Psi}+\tilde{\Psi} * \tilde{\Psi}=0
$$

where the lifted BRST charge is given by the natural expression

$$
\tilde{Q} \equiv \sum_{n}: c_{-n}\left(L_{n}^{\text {matter }}+L_{n}^{\text {aux }}+\frac{1}{2} L_{n}^{\text {ghost }}\right):
$$


The important physical property of $\tilde{\Psi}$ is that, while it should change the boundary conditions from $\mathrm{BCFT}_{0}$ to $\mathrm{BCFT}_{\Psi}$, as the original solution $\Psi$, it should however leave untouched the boundary conditions in the auxiliary BCFT ${ }^{\text {aux }}$. We expect that this can be systematically achieved by searching the lifted solution inside the Verma module of the identity operator of BCFT ${ }^{\text {aux }}$

$$
\tilde{\Psi}=\sum_{\{M\}} \Psi_{\{M\}} \otimes L_{-\{M\}}^{\text {aux }}|0\rangle^{\text {aux }} .
$$

Because of the $c=0$ nature of BCFT ${ }^{\text {aux }}$, the original solution $\Psi$ is just the coefficient of the $S L(2, R)$ vacuum

$$
\tilde{\Psi}=\Psi \otimes|0\rangle^{\text {aux }}+\sum_{\{M\} \neq \emptyset} \Psi_{\{M\}} \otimes L_{-\{M\}}^{\text {aux }}|0\rangle^{\text {aux }} .
$$

Given any CFT bulk primary of the form

$$
\mathscr{V}^{\alpha}(z, \bar{z})=c \bar{c} V^{\alpha}(z, \bar{z})
$$

where $V^{\alpha}$ is a purely matter primary of weight $\left(h_{\alpha}, h_{\alpha}\right)$, we can consider a formal bulk primary in $\mathrm{CFT}^{\text {aux }}, w^{\alpha}(z, \bar{z})$ of weight $\left(1-h_{\alpha}, 1-h_{\alpha}\right)$ with the property that

$$
\left\langle w^{\alpha}(0)\right\rangle_{\text {disk }}^{\mathrm{BCFT}^{\text {aux }}}=1, \quad \forall \alpha .
$$

Explicitly we can define BCFT ${ }^{\text {aux }}$ to be the tensor product of a free boson $Y$ with Dirichlet boundary conditions $(c=1)$ and a linear dilaton $\varphi$ with background charge $Q=\frac{1}{\sqrt{3}}$ with Neumann boundary conditions and $c=1-6 Q^{2}=-1$. In this case we can systematically take

$$
w^{\alpha}=e^{2 i \sqrt{1-h_{\alpha}} Y} e^{\frac{2 i}{\sqrt{3}} \varphi}
$$

which has weight $\left(1-h_{\alpha}, 1-h_{\alpha}\right)$ and satisfies (2.29), thanks to the Dirichlet conditions for $Y$ and the saturation of the background charge on the disk. Notice that, for $h_{\alpha}>1, w^{\alpha}$ is not normalizable in the auxiliary closed string Hilbert space, but still it has a well defined one-point function on the disk. Other choices of BCFT ${ }^{\text {aux }}$ are clearly possible. Now we tensor the compensator $w^{\alpha}$ with our original bulk field $\mathscr{V}^{\alpha}$ to form the weight zero primary

$$
\tilde{\mathscr{V}}^{\alpha}(z, \bar{z}) \equiv \mathscr{V}^{\alpha} \otimes w^{\alpha}(z, \bar{z})
$$

which is closed wrt to the lifted BRST charge (2.25)

$$
\tilde{Q}^{\tilde{V}^{\alpha}}=0,
$$

and satisfy

$$
\left\langle\tilde{\mathscr{V}}^{\alpha}(0) c(1)\right\rangle_{\mathrm{disk}}^{\mathrm{BCFT}_{0} \otimes \mathrm{BCFT}^{\mathrm{aux}}}=\left\langle\mathscr{V}^{\alpha}(0) c(1)\right\rangle_{\mathrm{disk}}^{\mathrm{BCFT}_{0}} .
$$

Given the lifted solution, making use of the Ellwood conjecture in the tensor theory, together with the assumption that the boundary conditions are non changed in BCFT ${ }^{\text {aux }}$, we can simply 
derive

$$
\begin{aligned}
& -4 \pi i\left\langle I\left|\tilde{\mathscr{V}}^{\alpha}(i)\right| \tilde{\Psi}-\tilde{\Psi}_{T V}\right\rangle^{\mathrm{BCFT}_{0}^{\prime}} \\
& =\left\langle\tilde{\mathscr{V}}^{\alpha}\left|c_{0}^{-}\right| \tilde{B}_{\tilde{\Psi}}\right\rangle \\
& =\left(\left\langlec \bar{c} V^{\alpha}\left|\otimes\left\langle w^{\alpha}\right|\right) c_{0}^{-}\left(\left|B_{\Psi}\right\rangle \otimes\left|B_{\text {aux }}\right\rangle\right)\right.\right. \\
& =\left\langle c \bar{c} V^{\alpha}\left|c_{0}^{-}\right| B_{\Psi}\right\rangle \times\left\langle w^{\alpha}(0)\right\rangle_{\text {disk }}^{\mathrm{BCFT}^{\mathrm{aux}}} \\
& =\left\langle c \bar{c} V^{\alpha}\left|c_{0}^{-}\right| B_{\Psi}\right\rangle=-2 n_{\Psi}^{\alpha} .
\end{aligned}
$$

Therefore we can get the coefficients of the Ishibashi states from the gauge invariant quantities

$$
n_{\Psi}^{\alpha} \equiv 2 \pi i\left\langle I\left|\tilde{\mathscr{V}}^{\alpha}(i)\right| \tilde{\Psi}-\tilde{\Psi}_{T V}\right\rangle .
$$

In the following we will test this proposal with analytic and numerical solutions.

\section{Analytic Solutions}

Here I illustrate the just outlined construction in an explicit case where Ellwood conjecture has been verified, and all OSFT computations have been done already. For definitness I focus on the simplest well-defined OSFT solutions corresponding to marginal deformations of the initial $\mathrm{BCFT}_{0}$, where the marginal current has regular OPE with itself, but the whole construction can be

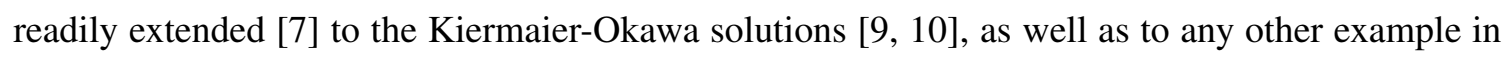
which the Ellwood invariant has been shown to analytically compute the tadpole shift, for example [ㄴ, [2]. An interesting example is given by the rolling tachyon marginal deformation generated by the marginal current $V=e^{X^{0}}$.

These solutions have been constructed in the $\mathscr{B}_{0}$-gauge in [[1]3, [4]] and extended to more general gauges in [155, ㅁ6]

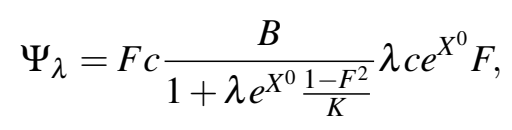

where $F=F(K)^{2}$ and $K, B, c$ are the familiar string fields [ㅁ], 미, 미, [20, ㅈ]], and $e^{X^{0}}$ is the insertion of the exactly marginal boundary operator : $e^{X^{0}}:(s)$ in the sliver frame.

Given an on-shell weight-zero primary closed string state $\mathscr{V}=c \bar{c} V^{(1,1)}$, the Ellwood invariant for this class of solutions has been computed in three different ways [22], 223, 24], and the result (with the $\mathrm{BCFT}_{0}$ contribution— given by the tachyon vacuum invariant—conveniently subtracted) is

$$
\begin{aligned}
& \left\langle I|\mathscr{V}(i)| \Psi-\Psi_{T V}\right\rangle=-\left\langle e^{-\lambda \int_{0}^{1} d s e^{X^{0}}(s)} \mathscr{V}(i \infty) c(0)\right\rangle_{C_{1}}^{\mathrm{BCFT}_{0}}
\end{aligned}
$$

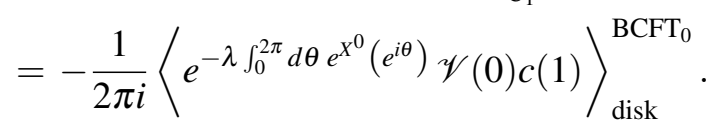

The nontrivial rearrangement of the $e^{X^{0}}$ insertions in the solution into a simple boundary interaction is a general consequence of the particular form of the solution and the string field $F(K)$, as discussed in [24, 25]].

\footnotetext{
${ }^{2}$ We assume the conditions $F(0)=1, F^{\prime}(0)<0$ and $F(\infty)=0$.
} 
This closed string tadpole is in fact closely related to the proper overlap of a closed string of the form $c \bar{c} V_{m}$ with the boundary state of $\Psi$

$$
\begin{aligned}
\left\langle e^{-\lambda \int_{0}^{2 \pi} d \theta e^{X^{0}}\left(e^{i \theta}\right)} c \bar{c} V_{m}^{(h, h)}(0) c(1)\right\rangle_{\mathrm{disk}}^{\mathrm{BCFT}} & =\frac{1}{2}\left\langle e^{-\lambda \int_{0}^{2 \pi} d \theta e^{X^{0}}\left(e^{i \theta}\right)}\left(c_{0}-\bar{c}_{0}\right) c \bar{c} V_{m}^{(h, h)}(0)\right\rangle_{\mathrm{disk}}^{\mathrm{BCFT}_{0}} \\
& \equiv \frac{1}{2}\left\langle B \Psi\left|c_{0}^{-}\right| c \bar{c} V_{m}^{(h, h)}\right\rangle
\end{aligned}
$$

where in the last line we have used the defining expression for the boundary state. Notice, that although this relation is trivially true for any matter operator, there is no gauge invariant observable in the OSFT defined on $\mathrm{BCFT}_{0}$ (with generic boundary conditions) that could give the LHS of (B.3)) for $h \neq 1$.

To overcome this difficulty we lift the solution to

$$
\mathrm{BCFT}_{0}^{\prime}=\mathrm{BCFT}_{0} \otimes \mathrm{BCFT}^{\mathrm{aux}}
$$

as outlined in the previous section. The simplest lift we can do is to simply replace the $\mathrm{BCFT}_{0}$ worldsheet with the $\mathrm{BCFT}_{0}$ ' one. This is operatorially achieved by the simple replacement

$$
\tilde{\Psi}=\Psi\left(K \rightarrow K+K^{\text {aux }}\right) .
$$

Consider now $\mathscr{V}^{(h)}=c \bar{c} V^{(h, h)}$, where $V^{(h, h)}$ is a weight $h$ level-matched primary of $\mathrm{BCFT}_{0}$. The state can be turned into a weight zero primary with a nonvanishing tadpole in $\mathrm{BCFT}_{0}^{\prime}$

$$
\tilde{\mathscr{V}}^{(h)}=\mathscr{V}^{(h)} e^{2 \sqrt{h-1} Y} e^{\frac{2 i \phi}{\sqrt{3}}} .
$$

Now we compute an Ellwood invariant in this slightly modified OSFT

$$
\begin{aligned}
& \left\langle I\left|\tilde{\mathscr{V}}^{(h)}(i)\right| \tilde{\Psi}-\tilde{\Psi}_{T V}\right\rangle=-\left\langle e^{-\lambda \int_{0}^{1} d s e^{X^{0}}(s)} \tilde{\mathscr{V}}^{(h)}(i \infty) c(0)\right\rangle_{C_{1}}^{\mathrm{BCFT}_{0}^{\prime}}
\end{aligned}
$$

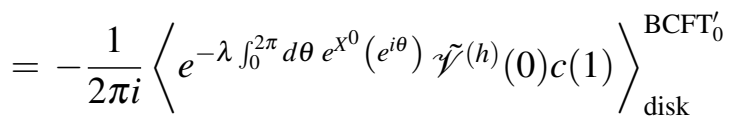

$$
\begin{aligned}
& =-\frac{1}{2 \pi i}\langle c \bar{c}(0) c(1)\rangle\left\langle e^{-\lambda \int_{0}^{2 \pi} d \theta e^{X^{0}}\left(e^{i \theta}\right)} V^{h, h}(0)\right\rangle^{\mathrm{BCFT}_{0}}\left\langle e^{2 \sqrt{h-1} Y} e^{\frac{2 i \phi}{\sqrt{3}}}(0)\right\rangle^{\mathrm{BCFT}^{\text {aux }}} \\
& =-\frac{1}{4 \pi i}\left\langle e^{-\lambda \int_{0}^{2 \pi} d \theta e^{X^{0}}\left(e^{i \theta}\right)}\left(c_{0}-\bar{c}_{0}\right) c \bar{c} V^{h, h}(0)\right\rangle_{\text {disk }}^{\mathrm{BCFT}_{0}},
\end{aligned}
$$

We thus found

$$
\begin{aligned}
\left\langle c \bar{c} V^{(h, h)}\left|c_{0}^{-}\right| B_{\Psi}\right\rangle & =-4 \pi i\left\langle I\left|\tilde{\mathscr{V}}^{(h)}(i)\right| \tilde{\Psi}-\tilde{\Psi}_{T V}\right\rangle \\
& =\left\langle c \bar{c} V^{h, h}(0)\left(c_{0}-\bar{c}_{0}\right) e^{-\lambda \int_{0}^{2 \pi} d \theta e^{X^{0}}\left(e^{i \theta}\right)}\right\rangle_{\text {disk }}^{\mathrm{BCFT}_{0}}
\end{aligned}
$$

Once this is true for any level-matched primary of $\mathrm{CFT}^{\text {matter }}$, it follows from the Virasoro gluing conditions that

$$
|B \Psi\rangle=e^{-\lambda \int_{0}^{2 \pi} d \theta e^{X^{0}}}\left|B_{0}\right\rangle,
$$


where $\left|B_{0}\right\rangle$ is the boundary state of $\mathrm{BCFT}_{0}$. Having a closed form expression for the boundary state we can now, for example, extract from it the energy momentum tensor of the solution, which turns out to be, see [3] for details,

$$
\begin{aligned}
\frac{T^{i j}\left(x^{0}\right)}{\operatorname{Vol}_{25}} & =-\frac{1}{1+2 \pi \lambda e^{x^{0}}} \delta^{i j}, \\
\frac{T^{00}\left(x^{0}\right)}{\operatorname{Vol}_{25}} & =1 .
\end{aligned}
$$

This is the usual energy-momentum tensor for a half S-brane exhibiting energy conservation and exponential decay for the pressure.

\section{Numerical Solutions}

Let's now test our method with a famous numerical solution, the Siegel-gauge lump, found by Moeller, Sen and Zwiebach [26]. They construct lump solutions along a compact direction $X$ with radius $R$ and such solutions are given up to level $L=3$ in terms of the towers

$$
\begin{aligned}
\left|T_{n}\right\rangle & =c_{1} \cos \left(\frac{n}{R} X(0)\right)|0\rangle, \\
\left|U_{n}\right\rangle & =c_{-1} \cos \left(\frac{n}{R} X(0)\right)|0\rangle, \\
\left|V_{n}\right\rangle & =c_{1} L_{-2}^{(X)} \cos \left(\frac{n}{R} X(0)\right)|0\rangle, \\
\left|W_{n}\right\rangle & =c_{1} L_{-2}^{\prime} \cos \left(\frac{n}{R} X(0)\right)|0\rangle, \\
\left|Z_{n}\right\rangle & =c_{1} L_{-1}^{(X)} L_{-1}^{(X)} \cos \left(\frac{n}{R} X(0)\right)|0\rangle,
\end{aligned}
$$

in the form

$$
|\Psi\rangle=\sum_{n \mid L \leq 3}\left(t_{n}\left|T_{n}\right\rangle+u_{n}\left|U_{n}\right\rangle+v_{n}\left|V_{n}\right\rangle+w_{n}\left|W_{n}\right\rangle+z_{n}\left|Z_{n}\right\rangle\right) .
$$

The Virasoro generators appearing in the expansion of the solution are purely matter, and are split according to the decomposition of the energy momentum tensor

$$
T_{c=26}^{\text {matter }}(z)=T_{c=1}^{(X)}(z)+T_{c=25}^{\prime}(z),
$$

in the two BCFT sectors

$$
\mathrm{BCFT}_{c=26}^{\text {matter }}=\mathrm{BCFT}_{c=1}^{X} \otimes \mathrm{BCFT}_{c=25}^{\prime} .
$$

Ghost degrees of freedom are spanned by ghost oscillators. For definiteness we consider the lump solution of the form (4.2) at radius $R=\sqrt{3}$. For this particular value, the reader can find the numerical results for the lump coefficients $\left(t_{n}, u_{n}, v_{n}, w_{n}, z_{n}\right)$ in table 3 of [26].

We will be interested especially in computing the energy density profile along the direction $X$ on which the lump is forming. These quantities can be easily obtained from generalized Ellwood invariants. What is needed is a lift for the numerical approximated solution. Since the solution is not turning on any primary along $\mathrm{BCFT}_{c=25}^{\prime}$, a simple lift is given by (4.2), with the replacement

$$
L^{\prime} \rightarrow L^{\prime}+L^{\text {aux }}
$$




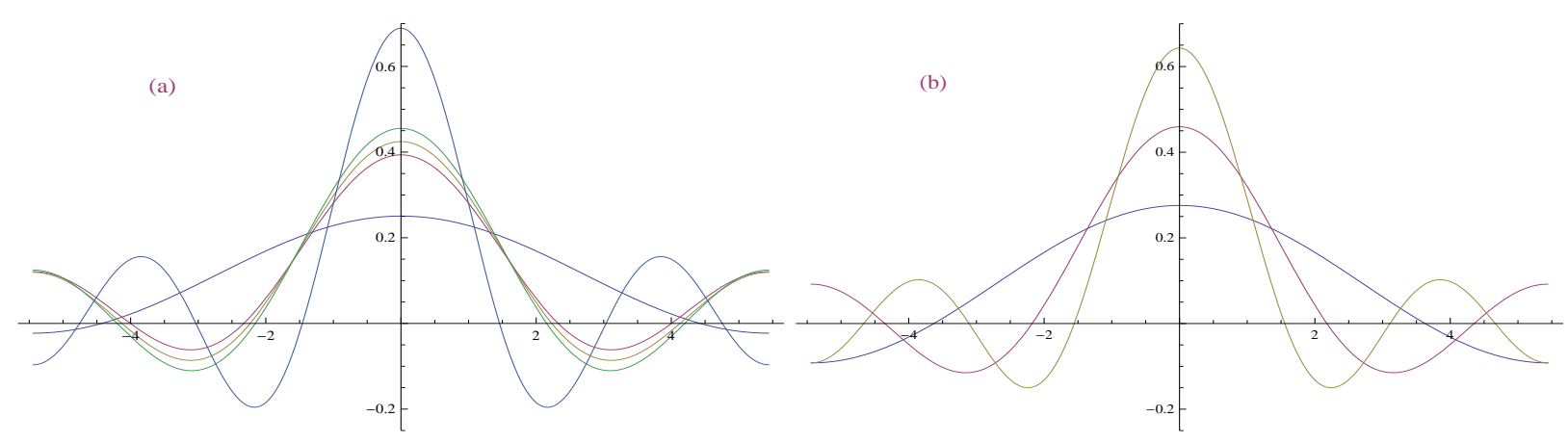

Figure 4.1: (a) Gauge-invariant energy density profile of the Siegel-gauge-lump at $R=\sqrt{3}$, as compared to the corresponding truncations of the delta function, $(b)$.

Equivalently, instead of tensoring with an auxiliary BCFT of $c=0$, we can just impose the Dirichlet boundary condition on an arbitrary space direction in $\mathrm{BCFT}_{c=25}^{\prime}$, say $Y \equiv X^{25}$, along which the solution does not change. Because of the universal structure in the $Y$-direction, the solution remains a solution and the coefficients of the Ishibashi states we compute are not affected by the new Dirichlet boundary conditions.

If we are interested in the energy profile, we have to compute the following generalized Ellwood invariants

$$
E_{n} \equiv-4 \pi i\left\langle E\left[c \bar{c} \partial X^{0} \bar{\partial} X^{0} e^{i \frac{n X}{R}+\frac{n Y}{R}}\right] \mid \Psi-\Psi_{T V}\right\rangle .
$$

The $n=0$ contribution is the mass of the brane configuration, normalized to 1 for a single lower dimensional D-brane; it is the coefficient of the Ishibashi state of the zero momentum graviton in the time-time direction. In terms of the momenta $E_{n}$, the energy density profile can be defined as a simple Fourier series

$$
E(x) \equiv T^{00}(x)=\frac{1}{\pi R}\left(\frac{1}{2} E_{0}+\sum_{n=1}^{\infty} E_{n} \cos \frac{n x}{R}\right) .
$$

If a solution describes a lower dimensional brane sitting at $x=0$, its energy density profile should be given by

$$
E(x)=\delta(x)=\frac{1}{\pi R}\left(\frac{1}{2}+\sum_{n=1}^{\infty} \cos \frac{n x}{R}\right) .
$$

Thus an exact lump solution sitting at $x=0$ will be characterized by

$$
E_{n}=1, \quad \forall n=0, \ldots, \infty \quad(\text { Exact Lump) }
$$

To compute these quantities we have used the conservation laws for the Ellwood Invariants, first derived in [27, 22], and we got the profile 4. la. To compare we plot the approximants of the delta function $\frac{1}{\pi R}\left(\frac{1}{2}+\sum_{n=1}^{N} \cos \frac{n}{R} x\right)$ for $N=1,2,3$, see figure $4 . \mathrm{d} \mathrm{b}$. It is also interesting to qualitatively compare with the known open-string-tachyon profile (given by $\sum_{n} t_{n} \cos \frac{n}{R} x$, see figure 4.2 ). It is apparent that in the 'closed-string' profile of figure 4.0 the higher harmonics play an essential role in localizing it to zero width, while this does not happen in the open string profile. This is a 


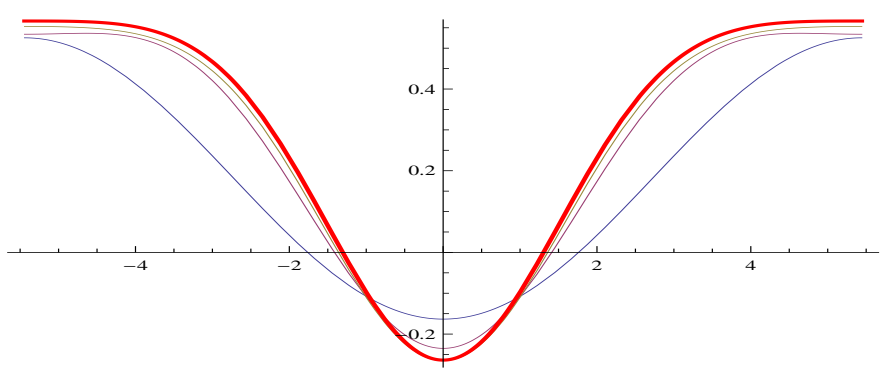

Figure 4.2: Traditional open string tachyon profile of the Siegel-gauge-lump: higher harmonics are suppressed in the Fock space and the open string profile is essentially unchanged as we increase the level.

consequence of the geometry of the identity string field, which effectively dresses the tachyon coefficients $t_{n}$ with $4 \frac{n^{2}}{R^{2}}$ thus amplifying the effect of higher harmonics. As it often happens, subleading contributions in the Fock space can have important sizable effects in observables.

Since our method worked well with a known solution, let us consider a more interesting solution, a multiple brane configuration, recently studied in [28]. The interest here is to show how our gauge invariant expression for the energy density can be used to measure the distance between the lower dimensional branes described by the solution. Suppose we have a solution $\Psi_{a}$ describing two D-branes on a circle of radius $R$, symmetric around the origin and at a distance $a(2 \pi R)$ from each other. The energy of the solution will be given by

$$
E_{0}=2
$$

meaning that we have two lower dimensional branes. But how does the number $a$ show up in the $E_{n}$ 's? The exact profile of a double lump configuration with separation $a(2 \pi R)$, centered around $\pi R$, is given by

$$
E_{(a)}(x)=\delta(x-\pi R(1-a))+\delta(x-\pi R(1+a))=\frac{1}{\pi R}\left(\frac{1}{2} E_{0}+\sum_{n=1}^{\infty} E_{n} \cos \frac{n x}{R}\right) .
$$

Integrating both sides against $\cos \frac{x}{R}$ gives

$$
\int_{0}^{2 \pi R} d x \cos \left(\frac{x}{R}\right) E_{(a)}(x)=-2 \cos (\pi a)=E_{1} .
$$

Thus, in the case of a two-lump solution, the invariant $E_{1}$ measures the distance between the two D-branes

$$
a_{1}=\frac{1}{\pi} \arccos \left(-\frac{E_{1}}{2}\right) .
$$

The arc-cosine is defined here in the standard branch $\arccos (0)=\frac{\pi}{2}$. The other branches would give the lengths of all the possible open strings stretching between the branes and wrapping the circle at the same time. Higher harmonics can also be used to compute the distance, and integrating (4.9) against $\cos \frac{n x}{R}$ we find

$$
E_{n}=2(-1)^{n} \cos (n \pi a)
$$




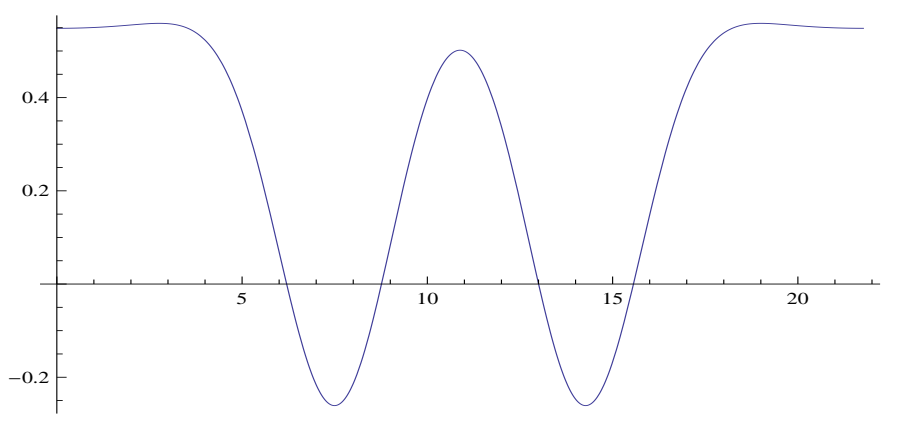

Figure 4.3: Open string tachyon profile of a two-lump solution obtained at $R=2 \sqrt{3}$ and level $L=(12,36)$.

Solving this equation for $a$ requires some care in choosing the correct branch of the arc-cosine. This must be done in such a way that the distance computed from any $E_{n}$ gives the same value $a_{1}$ as computed from $E_{1}$. The result can be written as

$$
a_{n}=(-1)^{p_{n}} \frac{1}{\pi n} \arccos \left((-1)^{n} \frac{E_{n}}{2}\right)+\frac{2\left[\frac{p_{n}+1}{2}\right]}{n}, \quad n>1
$$

where $[x]$ stands for integer part and the integer $p_{n}$ is uniquely chosen such that

$$
\frac{p_{n}}{n}<a_{1}<\frac{p_{n}+1}{n}
$$

Clearly for the exact solution $\Psi_{a}$ we should have

$$
a_{n}=a \equiv \text { Distance }, \quad \forall n \geq 1,
$$

which is a quite nontrivial constraint between the various $E_{n}$, which will be only approximatively satisfied at finite level. For generic multiple lump solutions, the relative distances between the various D-branes can be computed from the $E_{n}$ invariants along similar lines. Let us look at a particular example. At level $(12,36)$ we selected a double lump solution obtained at $R=2 \sqrt{3}$ which displays the open string tachyon profile shown in figure 4.3. Starting form the coefficients of the numerical solution we can compute the $E_{n}$ invariants and hence the distance at which the lower dimensional branes sit. Statistical treatment of the data (every harmonic gives an approximated distance, and all of them are statistically consistent, so that we can appropriately average between them, see [3] for details) gives

$$
a_{*}=0.299 \pm 0.001,
$$

Since we are 'measuring' a modulus of a BCFT in an unknown point of its moduli space via an approximate OSFT solution, we do not have a given value to compare with, but to appreciate to what extent $a_{*} \sim 0.3$ is consistent with the distance between the two D-branes described by the solution, we plot the energy profile of the solution including up to 6th harmonic against the corresponding truncation of a sum of two delta functions, at distance $a=0.3$, see figure 4.4. 

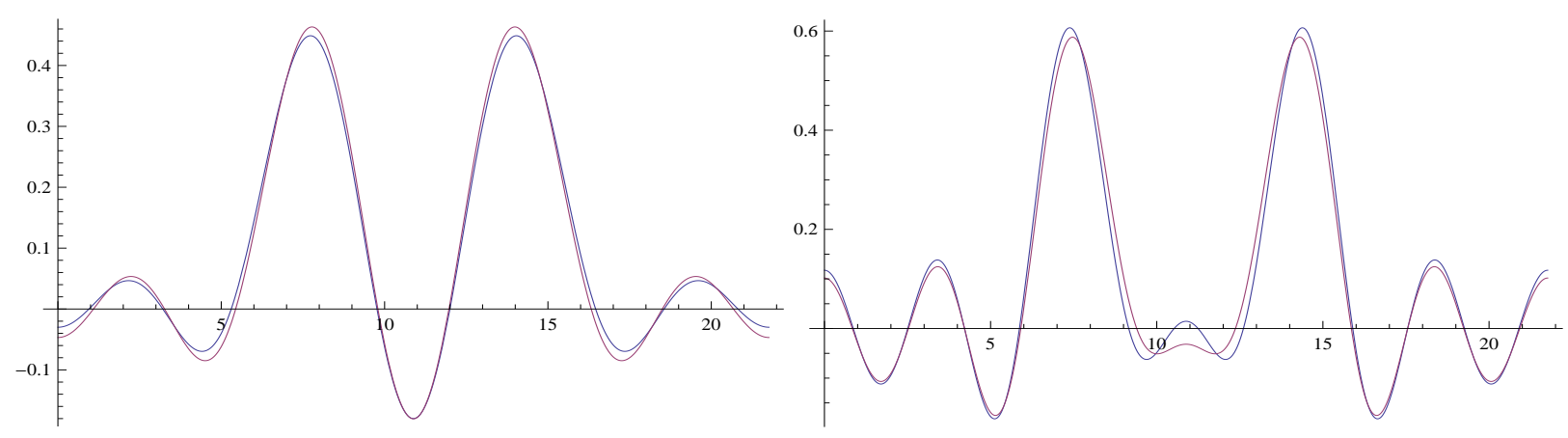

Figure 4.4: Plot of $\frac{1}{\pi R}\left(\frac{1}{2} E_{0}+\sum_{n=1}^{m} E_{n} \cos \frac{n x}{R}\right)$, in blue line, against the corresponding truncation of a sum of two delta functions, in magenta line, at a separation $\bar{a}_{*}=0.3$, for $m=4$ (left) and $m=6$ (right). Here $R=2 \sqrt{3}$ and the coefficients are obtained at level $L=(12,36)$. Notice how the profile of the truncated solution displays a slightly bigger effective distance than the 'exact', extrapolated one, as if the lumps were getting closer by increasing the level.

\section{Conclusions}

Making precise the relation between OSFT solutions and conformal boundary conditions is an important issue which gives a new perspective on the interplay between open and closed strings. In this talk, I have proposed a simple prescription which, under some natural assumptions, allows to compute the boundary state associated to a given OSFT solution, in a fairly simple way. The construction is gauge invariant and in all known cases it reproduces the expected boundary state. Quite importantly, the method is so simple that it allows to simply handle a large class of numerical solutions. In the future, it would be important to clarify some points

- Our construction starts with an appropriate definition of a lifted solution. Although in all known analytic and numerical examples we have today at our disposal, it is fairly obvious how to find such a lifted solution, it would be desirable to have a universal constructive procedure to systematically get a lifted solution (the simplest?) from a completely general given one. This would be needed, for example, for numerical D-instantons solutions.

- It would be interesting to make contact with the existing construction of the OSFT boundary state by Kiermaier, Okawa and Zwiebach, (KOZ) [ख2]. In particular it would be instructive to explore possible inter-relations in the construction of a generic lifted solution and the $\mathrm{KOZ}$ approach to the boundary state.

- The coefficients of the Ishibashi states should obey very constraining relations known as Cardy and sewing conditions, see e.g. [BO]. It is amazing that all known OSFT solutions give rise to boundary states which are consistent with this 'quantum' constraints. This suggests that the topological structure of the space of OSFT solutions somehow knows about modular invariance and open-closed factorization, although the theory is formulated on world sheets with the disk topology.

Understanding these issues will be useful to get quantitative and precise informations on the way string field theory describe the landscape and it will shed light on its hidden background 
independence. Perhaps not too optimistically, we are not so far to understand how closed string dynamics can be described with open string degrees of freedom, and thus to get a new powerful microscopic window in the gauge-gravity duality.

\section{References}

[1] E. Witten, "Noncommutative Geometry And String Field Theory," Nucl. Phys. B 268, 253 (1986).

[2] B. Zwiebach, "A Proof that Witten's open string theory gives a single cover of moduli space," Commun. Math. Phys. 142 (1991) 193. "Oriented open - closed string theory revisited," Annals Phys. 267 (1998) 193 [hep-th/9705241].

[3] M. Kudrna, C. Maccaferri, M. Schnabl and , "Boundary State from Ellwood Invariants," arXiv:1207.4785 [hep-th].

[4] J. A. Shapiro, C. B. Thorn and, "Closed String - Open String Transitions And Witten's String Field Theory," Phys. Lett. B 194 (1987) 43.

[5] A. Hashimoto, N. Itzhaki and , “Observables of string field theory,” JHEP 0201 (2002) 028 [hep-th/0111092].

[6] D. Gaiotto, L. Rastelli, A. Sen, B. Zwiebach and, "Ghost structure and closed strings in vacuum string field theory," Adv. Theor. Math. Phys. 6 (2003) 403 [hep-th/0111129].

[7] I. Ellwood, "The Closed string tadpole in open string field theory," JHEP 0808 (2008) 063 [arXiv:0804.1131 [hep-th]].

[8] N. Ishibashi, "The Boundary and Crosscap States in Conformal Field Theories," Mod. Phys. Lett. A 4 (1989) 251.

[9] M. Kiermaier and Y. Okawa, "Exact marginality in open string field theory: A General framework," JHEP 0911 (2009) 041 [arXiv:0707.4472 [hep-th]].

[10] E. Fuchs, M. Kroyter and R. Potting, "Marginal deformations in string field theory," JHEP 0709 (2007) 101 [arXiv:0704.2222 [hep-th]].

[11] L. Bonora, C. Maccaferri and D. D. Tolla, "Relevant Deformations in Open String Field Theory: a Simple Solution for Lumps," JHEP 1111 (2011) 107 [arXiv:1009.4158 [hep-th]].

[12] M. Murata and M. Schnabl, "Multibrane Solutions in Open String Field Theory," JHEP 1207 (2012) 063 [arXiv:1112.0591 [hep-th]].

[13] M. Schnabl, "Comments on marginal deformations in open string field theory," Phys. Lett. B $6 \mathbf{6 5 4}$ (2007) 194 [hep-th/0701248 [HEP-TH]].

[14] M. Kiermaier, Y. Okawa, L. Rastelli and B. Zwiebach, "Analytic solutions for marginal deformations in open string field theory," JHEP 0801 (2008) 028 [hep-th/0701249 [HEP-TH]].

[15] T. Erler, "Marginal Solutions for the Superstring," JHEP 0707 (2007) 050 [arXiv:0704.0930 [hep-th]].

[16] M. Kiermaier, Y. Okawa and P. Soler, "Solutions from boundary condition changing operators in open string field theory," JHEP 1103 (2011) 122 [arXiv:1009.6185 [hep-th]].

[17] M. Schnabl, "Analytic solution for tachyon condensation in open string field theory," Adv. Theor. Math. Phys. 10 (2006) 433 [arXiv:hep-th/0511286].

[18] Y. Okawa, "Comments on Schnabl's analytic solution for tachyon condensation in Witten's open string field theory," JHEP 0604 (2006) 055 [arXiv:hep-th/0603159]. 
[19] T. Erler, "Split string formalism and the closed string vacuum," JHEP 0705 (2007) 083 [arXiv:hep-th/0611200].

[20] T. Erler, "Split string formalism and the closed string vacuum. II,” JHEP 0705 (2007) 084 [arXiv:hep-th/0612050].

[21] M. Schnabl, “Algebraic solutions in Open String Field Theory - a lightning review," arXiv:1004.4858 [hep-th].

[22] I. Kishimoto, “Comments on gauge invariant overlaps for marginal solutions in open string field theory," Prog. Theor. Phys. 120 (2008) 875 [arXiv:0808.0355 [hep-th]].

[23] T. Noumi and Y. Okawa, "Solutions from boundary condition changing operators in open superstring field theory,” JHEP 1112 (2011) 034 [arXiv:1108.5317 [hep-th]].

[24] T. Erler and C. Maccaferri, “The Phantom Term in Open String Field Theory,” JHEP 1206 (2012) 084 [arXiv:1201.5122 [hep-th]].

[25] T. Erler and C. Maccaferri, "Connecting Solutions in Open String Field Theory with Singular Gauge Transformations,” JHEP 1204 (2012) 107 [arXiv:1201.5119 [hep-th]].

[26] N. Moeller, A. Sen, B. Zwiebach, "D-branes as tachyon lumps in string field theory,” JHEP 0008 (2000) 039. [hep-th/0005036].

[27] T. Kawano, I. Kishimoto and T. Takahashi, "Gauge Invariant Overlaps for Classical Solutions in Open String Field Theory,” Nucl. Phys. B 803 (2008) 135 [arXiv:0804.1541 [hep-th]].

[28] M. Kudrna, M. Schnabl, to appear

[29] M. Kiermaier, Y. Okawa and B. Zwiebach, "The boundary state from open string fields," arXiv:0810.1737 [hep-th].

[30] M. Gaberdiel, "Boundary conformal field theory and D-branes", Lectures given at the TMR network school on ŚNonperturbative methods in low dimensional integrable models ̌́, Budapest, 15-21 July 2003. http://www.phys.ethz.ch/ mrg/lectures2.pdf 